\title{
Existence de fécondations précoces dans le cycle biologique de Tomicus piniperda L. (Coleoptera Scolytidae) en forêt d'Orléans
}

\author{
Jean-Louis JANIN (*) \& François LIEUTIER \\ avec la collaboration technique de Jacques Garcia
}

I.N.R.A., Station de Zoologie forestière, Centre de Recherches d'Orléans, Ardon, F 45160 Olivet

RÉSUMÉ L'examen de la spermathèque de femelles adultes de Tomicus piniperda à différentes phases de leur matura-
tion montre qu'aucune jeune femelle n'est fécondée au moment de l'envol vers les pousses. En revanche, 10 à
$15 \mathrm{p} .100$ sont fécondées pendant la phase de nutrition dans les pousses. Les fécondations continuent pendant
l'hivernation à la base des arbres. A l'essaimage de printemps, 50 p. 100 des femelles sont déjà fécondées.

Mots clés additionnels : Fécondation, accouplement, maturation, France. Orléans (France).

The spermatheca of adult females of Tomicus piniperda was examined in detail for various stages of maturation of the insect. At the time when young females were taking flight before maturation feeding, none of them had mated. On the other hand, 10 to $15 \%$ of the females mated during maturation feeding in the shoots. Mating continued during over-wintering. At the time of swarming, $50 \%$ of females had already mated. Accordingly, in this insect, mating did not take place at a precise time in adult life (spring attack), but rather occurred progressively. This might be the case in most members of the Scolytidae.

Additional key words : Fertilization, mating, maturation, France.

\section{INTRODUCTION}

Tomicus piniperda L., coléoptère Scolytidae, d'une grande importance économique en Europe, présente en France une génération par an, et peut se développer sur toutes les espèces de pin. A la fin de l'hiver (fin février, début mars en Forêt d'Orléans), les adultes ayant hiverné dans l'écorce à la base des troncs s'envolent pour coloniser de nouveaux arbres. L'espèce est monogame, la femelle pénètre à l'intérieur du végétal hôte en creusant un orifice à travers l'écorce, puis le mâle la rejoint. D'après les données classiques, la fécondation a lieu à cette étape du cycle. La femelle creuse ensuite une galerie dans le liber et y dépose ses œufs. Les larves se nourrissent aux dépens du liber. Après la mue imaginale, les adultes immatu-

$\left(^{*}\right)$ Adresse actuelle : I.N.R.A.-C.N.R.S., Laboratoire de Neurobiologie comparée des Invertébrés, Route de la Guyonnerie, 91440 Bures-sur-Yvette. res émergent de l'arbre et s'envolent pour poursuivre leur maturation dans la moelle de jeunes rameaux de pin dans le courant de l'été. Cette activité crée des dégâts importants : les pousses flétrissent et tombent au sol. Il semble que les insectes puissent utiliser successivement plusieurs pousses. A la fin de l'automne, ils migrent vers leurs sites d'hivernation.

Ce type classique, apparemment simple, se complique par l'existence de générations-sœurs. Après une première ponte, les parents peuvent en effet réémerger et les femelles repénétrer dans les arbres pour y déposer une nouvelle ponte. Le nombre de générationssœurs ainsi produites n'est pas clairement établi. Il est possible aussi qu'une nouvelle alimentation ait lieu dans les pousses entre 2 pontes successives (CHARARAS, 1962 ; JAMIN, 1977 ; VALLET, 1981).

La connaissance du nombre et de l'importance des générations-sœurs est fondamentale pour décrire la dynamique des populations de l'espèce. Pour fixer l'âge des individus, un critère de distinction des générations parentales (issues de l'année précédente) et des 
nouvelles générations (issues de l'année en cours) pourrait être l'état fécondé ou non des femelles, mais cet éventuel critère ne peut être valable qu'à plusieurs conditions : il faut que les spermatozoïdes se conservent dans les voies génitales femelles un temps très long après la fécondation. Il faut vérifier que la fécondation n'a lieu que lors de l'attaque des arbres juste après l'essaimage de printemps. Or, en ce qui concerne cette seconde condition, des observations réalisées sur d'autres espèces, en particulier sur Dendroctonus ponderosae Hopkins (Mc CAMBRIDGE, 1969) et sur Ips grandicollis Eichoff font état de fécondations plus précoces (WITANACHCHI, 1980). Nous nous proposons donc, dans la présente note, de situer la place de la fécondation dans le cycle biologique de $T$. piniperda.

\section{MATÉRIEL ET MÉTHODES}

Le critère de fécondation utilisé a été la présence de spermatozoïdes dans les voies génitales femelles. L'observation a eu lieu au microscope après écrasement de l'organe débarrassé du tissu adipeux. Les spermatozoïdes se trouvent généralement dans la pièce chitinisée de la spermathèque, dans le réservoir annexé à cette pièce et éventuellement dans le canal aboutissant à la pièce chitinisée (fig. 1).

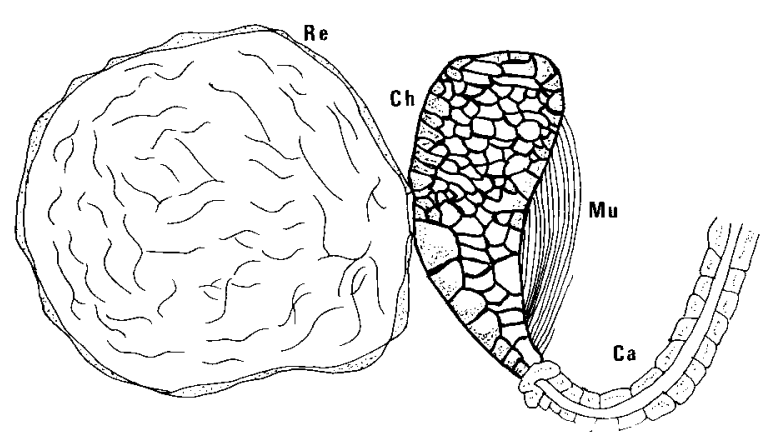

Figure 1

Spermathèque et pièces annexes de T. piniperda. $\mathrm{Ca}=$ canal; $c h=$ pièce chinitisée $; M u=$ muscle $;$ Re $=$ réservoir .

Spermatheca and accessory parts of $\mathrm{T}$. piniperda : $\mathrm{Ca}=$ duct ; $c h=$ chitinized piece $; M u=$ muscle $; \operatorname{Re}=$ reservoir.

Les insectes ont été disséqués à différentes phases de leur cycle durant leur vie adulte. La collecte des adultes immatures et des adultes ayant déjà pondu a été effectuée à partir de rondins naturellement infestés et placés en cage d'émergence. Les adultes en nutrition de maturation dans les pousses ont été extraits par ouverture de pousses infestées, tombées au sol dans la nature ou coupées sur un pin placé sous cage grillagée et infesté expérimentalement (cf. infra). Les insectes en hivernation ont été collectés par écorçage de la base des troncs, en janvier et en mars. Les individus à l'essaimage de printemps ont été capturés par piégeage à la glu : les bandes collantes avaient été fixées à intervalles réguliers sur le tronc d'arbres abattus peu avant la période de vol de l'insecte et constituant ainsi une masse attractive.
La nutrition des adultes dans les pousses a pu être, en outre, réalisée expérimentalement. Une cage grillagée a été placée autour d'un jeune pin de 7 ans, dans la nature. De jeunes adultes immatures, ainsi que des parents en réémergence (individus ayant déjà pondus) ont été lâchés dans l'enceinte en juin. Une technique de marquage, mise au point au préalable a permis de distinguer les 2 catégories d'insectes (LIEUTIER et al., 1986). A l'automne, les pousses attaquées ont été coupées sur l'arbre puis ouvertes.

\section{RÉSULTATS ET INTERPRÉTATION}

Le taux de fécondation croît régulièrement de la mue imaginale à la réémergence (fig. 2).

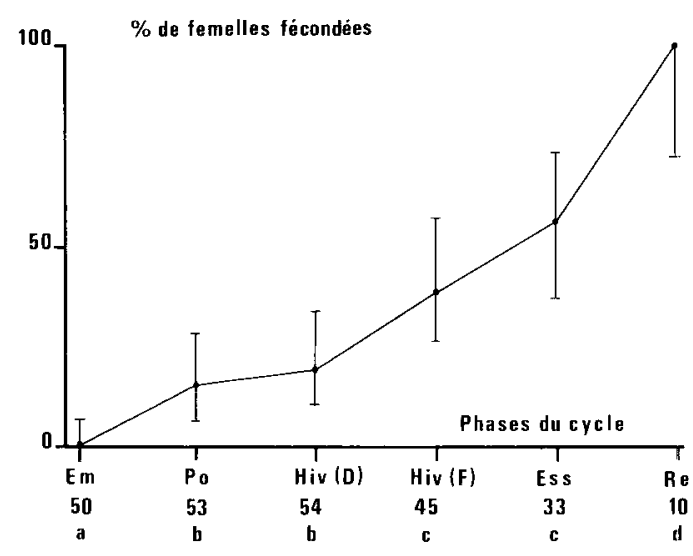

Figure 2

Evolution du pourcentage de femelles fécondées au cours du cycle biologique de $\mathrm{T}$. piniperda.

$E_{m}$ : émergence après la mue imaginale. Po: nutrition de maturation dans les pousses. Hiv. (D) : début de la phase d'hivernation. Hiv. (F) : fin de la phase d'hivernation. Ess : essaimage de printemps avant la ponte. Re : réémergence après la ponte.

Les chiffres indiqués en abscisse correspondent au nombre de femelles disséquées à chaque phase du cycle. Les différences entre les pourcentages de fécondation de 2 phases sont significatives (au seuil $5 \mathrm{p} .100)$ lorsque celles-ci sont affectées de lettres différentes (test t). Chaque valeur est accompagnée de son intervalle de confiance (barre verticale).

Variation in the percentage of mated females during the life cycle of $\mathrm{T}$. piniperda.

$E_{m}$ : flight at the beginning of adult stage; Po : shoot-feeding; Hiv. (D) : beginning of the overwintering period; Hiv. $(F)$ : end of the overwintering period; Ess : swarming before oviposition; Re 2nd swarming after oviposition.

Data in abscissa are numbers of dissected females. Percentages mating in the 2 periods significantly different (at $95 \%$ level) when these periods are indicated with different characters (T test). Each value is given with its confidence interval.

\section{A. Emergence après la mue imaginale et envol vers les pousses}

A ce stade, il est possible de distinguer sans difficulté les individus de la nouvelle génération, par leur coloration brun-clair, des parents en réémergence dont la pigmentation est brun-foncé à noire. Les jeunes femelles à l'émergence sont toutes vierges. Le nombre d'individus examinés (50 femelles) paraît suffisant pour admettre que la fécondation n'est pas encore réalisée lors de l'émergence post mue imaginale et de l'envol des insectes vers les pousses. 
TABLEAU 1

Expérimentation sur pin en cage grillagée.

Experiment with the caged pine.

\begin{tabular}{|c|c|c|c|c|c|c|}
\hline & \multicolumn{3}{|c|}{$\begin{array}{l}\text { Insectes introduits } \\
\text { dans la cage (juillet) }\end{array}$} & \multicolumn{3}{|c|}{$\begin{array}{c}\text { Insectes récupérés } \\
\text { dans les pousses (novembre) }\end{array}$} \\
\hline & $\begin{array}{l}\text { Nombre } \\
\text { total } \\
\text { d'individus }\end{array}$ & $\begin{array}{c}\text { Nombre de } \\
\text { femelles } \\
\text { disséquées }\end{array}$ & $\begin{array}{l}\text { Pourcentage } \\
\text { de femelles } \\
\text { fécondées }\end{array}$ & $\begin{array}{c}\text { Nombre } \\
\text { total } \\
\text { d'individus }\end{array}$ & $\begin{array}{l}\text { Nombre de } \\
\text { femelles } \\
\text { disséquées }\end{array}$ & $\begin{array}{c}\text { Pourcentage } \\
\text { de femelles } \\
\text { fécondées }\end{array}$ \\
\hline $\begin{array}{l}\text { Descendants } \\
\text { (marqués) }\end{array}$ & 379 & 53 & 0 & 64 & 50 & 9 \\
\hline $\begin{array}{l}\text { Parents } \\
\text { (non marqués) }\end{array}$ & 91 & 32 & 100 & 0 & - & - \\
\hline
\end{tabular}

\section{B. Maturation dans les pousses}

Le taux de fécondation des femelles récoltées dans les pousses de pin tombées au sol en novembre est faible mais significativement différent de zéro. Ces femelles fécondées peuvent être soit des parents ayant déjà pondu et étant retourné s'alimenter, soiţ de jeunes femelles en première nutrition de maturation et qui se seraient donc accouplées dans les pousses. Les résultats de l'expérimentation sous cage avec des individus marqués (tabl. 1) montrent toutefois qu'aucun individu parental n'est retrouvé dans les pousses. On ne peut donc pas conclure, en ce qui concerne la rematuration des parents. En revanche, parmi les descendants retrouvés dans les pousses, 9 p. 100 de femelles sont fécondées. En outre, les pourcentages de fécondation dans les pousses coupées sur l'arbre de la cage grillagée ( 9 p. 100) et dans celles ramassées au sol dans la nature $(15$ p. 100$)$ sont proches. Il est donc permis de penser que, dans la nature, une certaine proportion des individus immatures peut être fécondée au cours de la nutrition de maturation dans les pousses. Cette fécondation pourrait avoir lieu lors des rencontres entre individus au cours des passages d'une pousse à l'autre.

L'absence de parents dans les pousses en novembre peut s'expliquer, soit par une forte mortalité, soit par une alimentation de rematuration de très courte durée. On peut aussi toutefois s'interroger sur l'existence de cette nutrition de rematuration au niveau des pousses.

\section{Hivernation}

Les individus fécondés au début de l'hivernation (fig. 2) comportent certainement, pour une part au moins, des jeunes adultes qui se sont accouplés dans les pousses. On ne peut écarter totalement l'hypothèse qu'il s'y ajoute quelques parents hivernant une seconde fois bien que ce phénomène n'ait jamais été rapporté. Toutefois, le nombre de ces derniers doit cependant être extrêmement faible puisque le pourcentage de fécondation à cette époque est voisin de celui observé dans les pousses.

On observe ensuite une augmentation du taux de fécondation au cours de l'hivernation, jusqu'à l'essaimage de printemps où la moitié des femelles s'envo- lent déjà fécondées. Cette augmentation est statistiquement significative. On est donc conduit à admettre l'existence de fécondations dans les sites d'hivernation. Ces fécondations doivent intervenir sans doute lors des phases de réchauffement hivernal dans les zones éclairées du biotope ; on sait, en effet, que les températures sous-corticales peuvent atteindre, l'hiver, sur les faces ensoleillées des arbres, des valeurs compatibles avec une certaine activité des insectes. Les fécondations doivent alors être facilitées par le fréquent groupement des individus, soit dans une même galerie d'hivernation, soit dans des galeries différentes mais situées dans une même fente d'écorce.

\section{CONCLUSION}

Chez Tomicus piniperda, la fécondation ne semble pas intervenir avant la phase de nutrition dans les pousses. Elle débute pendant la nutrition, se poursuit pendant l'hiver, et s'achève au printemps après installation des insectes sur le fût des arbres attaqués.

Il n'est donc pas possible d'utiliser l'état fécondé ou non fécondé pour distinguer les femelles appartenant à différentes générations.

Néanmoins, ces résultats sont intéressants car ils montrent que la fécondation n'a pas lieu à un moment déterminé de la vie des adultes (en particulier pas uniquement lors de l'attaque des arbres au printemps) mais est au contraire progressive depuis la phase de nutrition de maturation. Mc CAMBRIDGE (1969) et WITANACHCHI (1980) avaient déjà observé des fécondations précoces respectivement chez un Dendroctonus et un Ips ce qui, avec le genre Tomicus représente 3 groupes de Scolytidae dont la position systématique est très diverse. On sait, en outre, que chez Dendroctonus micans, espèce à la biologie encore très différente, les femelles sont la plupart du temps fécondées avant l'émergence (CHARARAS, 1962). On est donc amené à suggérer que le phénomène de fécondation précoce doit être très répandu dans la famille des Scolytidae. Il serait à ce sujet intéressant de le rechercher chez des espèces françaises autres que $T$. piniperda et $D$. micans. 


\section{REFFERENCES BIBLIOGRAPHIQUES}

Chararas C., 1962. Etude biologique des Scolytides des Conifères. Lechevalier Ed., Paris, 556 p.

Jamin J. K., 1977. Contribution à l'étude du dépérissement provoqué par les coléoptères Scolytidae sur pin sylvestre en région Centre. Mémoire E.N.I.T.E.F., 84 p., Nogent-sur-Vernisson.

Lieutier F., Ham R., Ham M. C., Garcia J., 1986. Une méthode de marquage individuel des coléoptères scolytides pour les études de laboratoire. Agronomie, 6, 773-776.

Mc Cambridge W. F., 1969. Spermatozoa in unemerged female mountain pine beetle Dendroctonus ponderosae Hopkins. Proc. ent. Soc. Ont., 100, 168-170.

Vallet E., 1981. Etude du dépérissement du pin sylvestre en région Centre et des principaux ravageurs Scolytides associés: Tomicus piniperda, Ips sexdentatus, Ips acuminatus (Col. Scolytidae). Thèse de $3^{e}$ cycle, Université d'Orléans-la-Source. U.E.R. Sciences Fondamentales et Appliquées, $143 \mathrm{p}$

Witanachchi J. P., 1980. Evidence for pre-emergence mating among mature progeny of Ips grandicollis (Eichhof). J. Austr. ent. Soc., $19,93-100$ 